\title{
Long-chain polyunsaturated fatty acids and inflammation
}

\author{
Philip C. Calder
}

Institute of Human Nutrition, School of Medicine, University of Southampton, Southampton, UK

Abstract

Although part of the normal host response to infection or injury, inflammation is involved in many pathological conditions and disease states. Most interest in the influence of fatty acids on inflammatory processes has centred on the opposing actions of n- 6 and n-3 polyunsaturated fatty acids (PUFAs). The n-6 PUFA arachidonic acid gives rise to the eicosanoid family of inflammatory mediators (prostaglandins, leukotrienes and related metabolites) and through these regulates the activities of inflammatory cells, the production of inflammatory cytokines, etc. Consumption of long-chain n-3 PUFAs [eicosapentaenoic acid (EPA) and docosahexaenoic acid (DHA)] decreases the amount of arachidonic acid in inflammatory cell membranes and so available for eicosanoid production. Thus, consumption of long-chain n-3 PUFAs results in decreased production of eicosanoids from arachidonic acid. EPA acts as an alternative substrate for eicosanoid synthesis giving rise to mediators that are less potent than the analogues produced from arachidonic acid. EPA and DHA give rise to newly discovered families of mediators termed E- and Dresolvins, respectively, which have anti-inflammatory and inflammation-resolving actions. In addition to this range of effects, long-chain n-3 PUFAs affect cell-signalling processes and gene expression in inflammatory cells, resulting in decreased expression of inflammatory cytokines and adhesion molecules. Such long-chain n-3 PUFA-induced effects may be of importance in protecting against the development of and lowering the severity of acute and chronic inflammatory conditions. There is good evidence for the efficacy of long-chain n-3 PUFAs in rheumatoid arthritis, with less strong evidence in other inflammatory conditions. The precursor n-3 PUFA, $\alpha$-linolenic acid, exerts some anti-inflammatory effects at very high intakes, perhaps reflecting the need for its conversion to EPA to be effective.

Keywords: arachidonic acid; cytokine; eicosanoid; fish oil; inflammation; macrophage

Abbreviations: COX: cyclooxygenase; DHA: docosahexaenoic acid; EPA: eicosapentaenoic acid; HETE: hydroxyeicosatetraenoic acid; HPETE: hydroperoxyeicosatetraenoic acid; ICAM-1: intercellular adhesion molecule-1; I $\kappa$ : inhibitory subunit of $N F-\kappa B$; IL: interleukin; LOX: lipoxygenase; LT: leukotriene; NF- $\kappa B$ : nuclear factor- $\kappa B ; P G$ : prostaglandin; PUFA: polyunsaturated fatty acid; TNF: tumour necrosis factor; TX: thromboxane; VCAM-1: vascular cell adhesion molecule-1.

\section{Inflammation in health and disease}

I nflammation is part of the body's immediate response to infection or injury. It is typified by redness, swelling, heat and pain. These occur as a result of increased blood flow, increased permeability across blood capillaries, which allows large molecules (e.g. complement, antibodies, cytokines) to leave the bloodstream and cross the endothelial wall, and increased movement of leukocytes from the bloodstream into the surrounding tissue. Inflammation functions to begin the immunological process of elimination of invading pathogens and toxins and to repair damaged tissue. These responses must be ordered and controlled. The movement of cells into the inflammatory/infected site is induced by the up-regulation of adhesion molecules such as intercellular adhesion molecule-1 (ICAM1), vascular cell adhesion molecule-1 (VCAM-1) and E-selectin on the surface of endothelial cells allowing leukocyte binding and subsequent diapedesis. The earliest cells appearing at inflamed sites are granulocytes, with monocyte/macrophages and lymphocytes appearing later. Granulocytes and monocyte/macrophages are involved in pathogen killing, in clearing up cellular and tissue debris, and in tissue repair. The activity of these cells is induced by certain triggers, including components of bacterial cell walls. One such component is bacterial endotoxin (also known as lipopolysaccharide), part of the cell wall of Gram-negative bacteria. Endotoxin 
can directly activate monocyte/macrophages, inducing them to form cytokines such as tumour necrosis factor (TNF)- $\alpha$, interleukin (IL)-1, IL-6 and IL-8, eicosanoids, such as prostaglandin (PG) $\mathrm{E}_{2}$, nitric oxide, matrix metalloproteinases and other mediators. Endotoxin also induces adhesion molecule expression on the surface of endothelial cells and leukocytes. The cytokines produced by monocyte/macrophages also serve to regulate the whole-body response to infection and injury. Thus, inflammation and the inflammatory response are part of the normal, innate immune response. Inflammatory mediators also provide a link between the innate and acquired immune responses.

Although inflammation is a normal response, when it occurs in an uncontrolled or inappropriate manner excessive damage to host tissues and disease can ensue. Such uncontrolled or inappropriate inflammatory responses are characterized by hyperexpression of endothelial and leukocyte adhesion molecules, appearance of soluble forms of adhesion molecules in the circulation, sequestration of leukocytes to sites where they are not usually found, production of inflammatory mediators and damage to host tissues. High levels of TNF- $\alpha$, IL-1 $\beta$ and IL6 are particularly destructive and are implicated in causing some of the pathological responses that occur in endotoxic shock, in adult respiratory distress syndrome and in chronic inflammatory diseases such as rheumatoid arthritis and inflammatory bowel diseases. Chronic overproduction of TNF- $\alpha$ and IL- 1 can cause muscle wasting and loss of bone mass and may account for alterations in body composition and tissue loss seen in inflamma-

Table I. Examples of diseases or conditions that involve an inflammatory component

$\begin{array}{ll}\text { Rheumatoid arthritis } & \text { Multiple sclerosis } \\ \text { Crohn's disease } & \text { Neurodegenerative disease of ageing } \\ \text { Ulcerative colitis } & \text { Atherosclerosis } \\ \text { Lupus } & \text { Acute cardiovascular events } \\ \text { Type I diabetes } & \text { Obesity } \\ \text { Cystic fibrosis } & \text { Metabolic syndrome } \\ \text { Childhood asthma } & \text { Major surgery } \\ \text { Adult asthma } & \text { Trauma and critical illness } \\ \text { Chronic obstructive } & \text { Acute respiratory distress syndrome } \\ \text { pulmonary disease } & \text { Cancer cachexia } \\ \text { Allergic disease } & \text { Some cancers } \\ \text { Atopic dermatitis } & \\ \text { Psoriasis } & \end{array}$

tory diseases. As well as its clear and obvious association with classic inflammatory diseases, inflammation is now recognized to play an important role in the pathology of other diseases such as cardiovascular disease. Table 1 lists some diseases and conditions that are recognized to have an inflammatory component.

\section{The link between fatty acids and inflammation: arachidonic acid and eicosanoids}

The eicosanoid family of inflammatory mediators is generated from 20-carbon polyunsaturated fatty acids (PUFAs) liberated from cell-membrane phospholipids. The membrane phospholipids of inflammatory cells taken from humans consuming Western-type diets typically contain approximately $20 \%$ of fatty acids as the n- 6 PUFA arachidonic acid (1-7). In contrast, the proportions of other 20-carbon PUFAs such as the n-6 PUFA di-homo$\gamma$-linolenic acid and the n-3 PUFA eicosapentaenoic acid (EPA) are typically about 2 and $<1 \%$ of fatty acids, respectively $(4,6)$. Thus, arachidonic acid is usually the dominant substrate for eicosanoid synthesis. Eicosanoids include PGs, thromboxanes (TXs), leukotrienes (LTs) and hydroxyeicosatetraenoic acids (HETEs). Arachidonic acid in cellmembrane phospholipids can be mobilized by various phospholipase enzymes, most notably phospholipase $\mathrm{A}_{2}$, and the free acid can subsequently act as a substrate for the enzymes that synthesize eicosanoids (Fig. 1). Metabolism by cyclooxygenase (COX) enzymes gives rise to the 2series PGs and TXs. COX-2 is induced in inflammatory cells as a result of stimulation and is responsible for the markedly elevated production of PGs that occurs upon cellular activation. Monocytes and macrophages produce large amounts of $\mathrm{PGE}_{2}$ and $\mathrm{PGF}_{2}$, neutrophils produce moderate amounts of $\mathrm{PGE}_{2}$ and mast cells produce $\mathrm{PGD}_{2}$. Metabolism of arachidonic acid by the 5-lipoxygenase (5-LOX) pathway gives rise to hydroxy and hydroperoxy derivatives (5-HETE and 5-HPETE, respectively), and the 4-series LTs, $\mathrm{LTA}_{4}, \mathrm{~B}_{4}, \mathrm{C}_{4}$, $\mathrm{D}_{4}$ and $\mathrm{E}_{4}$. Neutrophils, monocytes and macrophages produce $\mathrm{LTB}_{4}$, while $\mathrm{LTC}_{4}, \mathrm{D}_{4}$ and $\mathrm{E}_{4}$ tend to be produced by mast cells, basophils and eosinophils.

$\mathrm{PGE}_{2}$ has a number of proinflammatory effects including inducing fever, increasing vascular permeability and vasodilatation, and enhancing pain and oedema caused by other agents. $\mathrm{PGE}_{2}$ has been 


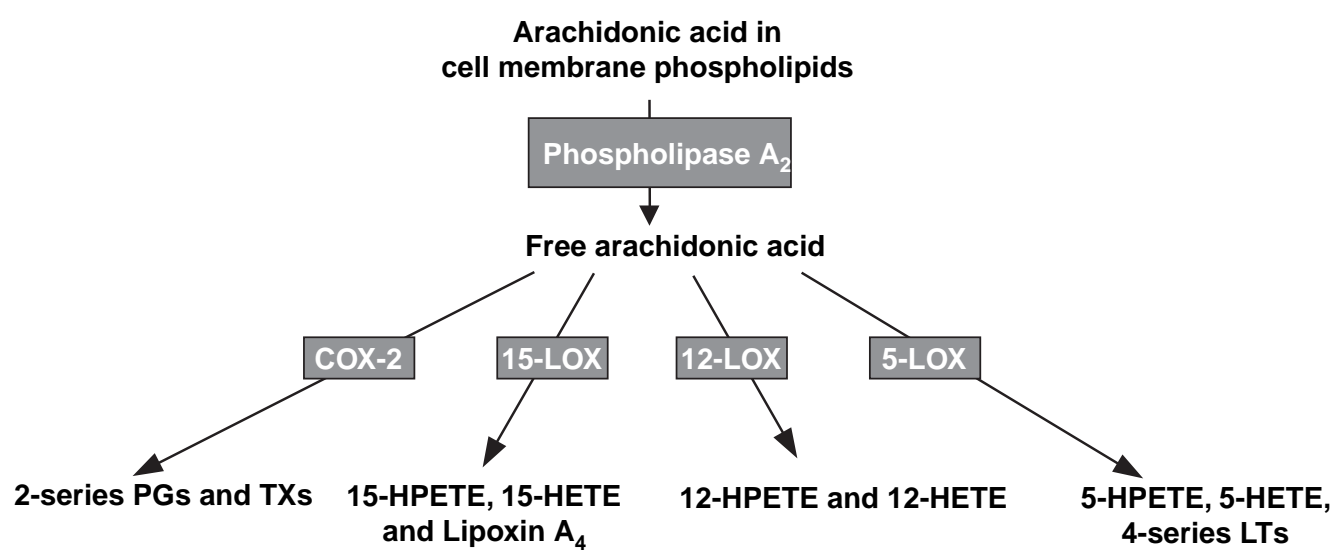

Fig. 1. General scheme of eicosanoid synthesis from arachidonic acid. Arachidonic acid is released from inflammatory cell membrane phospholipids by the action of phospholipases, especially phospholipase $\mathrm{A}_{2}$, and the free arachidonic acid acts as a substrate for cyclooxygenase (COX) and lipoxygenase (LOX) enzymes, giving rise to prostaglandins (PGs), thromboxanes (TXs), hydroperoxyeicosatetraenoic acids (HPETEs), hydroxyeicosatetraenoic acids (HETEs) and leukotrienes (LTs). These mediators have various roles in the inflammatory process.

shown to induce COX-2 mRNA expression in cultured fibroblasts, and so to up-regulate its own production, and to induce production of the inflammatory cytokine IL-6 by macrophages (8). $\mathrm{LTB}_{4}$ increases vascular permeability, is a potent chemotactic agent for leukocytes, induces release of lysosomal enzymes, and enhances generation of reactive oxygen species and production of inflammatory cytokines such as TNF- $\alpha$, IL-1 and IL-6. The cysteinyl-LTs $\left(\mathrm{LTC}_{4}, \mathrm{D}_{4}\right.$ and $\left.\mathrm{E}_{4}\right)$ are bronchoconstrictors, increase vascular permeability and promote hypersensitivity. In inflammatory conditions increased rates of production of arachidonic acid-derived eicosanoids occur and elevated levels of these eicosanoids are observed in blood and tissues from patients with acute and chronic inflammatory conditions. Despite the ongoing emphasis on the proinflammatory effects of arachidonic acid-derived eicosanoids, some of these mediators, for example lipoxin $\mathrm{A}_{4}$, are actually anti-inflammatory (9). Indeed, recent studies have highlighted an anti-inflammatory action of $\mathrm{PGE}_{2}$ which was shown to inhibit 5-LOX and so decrease production of 4-series LTs and to induce 15-LOX, so promoting the formation of anti-inflammatory lipoxins (9). Thus, $\mathrm{PGE}_{2}$ exhibits both proinflammatory and anti-inflammatory actions.

\section{Arachidonic acid and inflammatory mediator production}

Animal feeding studies have shown a strong positive relationship between the amount of arachidonic acid in inflammatory cells and the ability of those cells to produce eicosanoids such as $\mathrm{PGE}_{2}$ (10). In turn, the amount of arachidonic acid in inflammatory cells can be increased by including it in the diet $(10,11)$. The amount of arachidonic acid in inflammatory cells may also be influenced by the dietary intake of its precursor, linoleic acid, although the range of linoleic acid intake over which this relationship occurs has not been defined for humans. Increasing linoleic acid intake by $6.5 \mathrm{~g}$ per day in humans habitually consuming $10-15 \mathrm{~g}$ per day did not alter the arachidonic acid content of blood mononuclear cells (6). Nevertheless, the role of arachidonic acid as a precursor for the synthesis of eicosanoids indicates the potential for dietary n-6 PUFAs (linoleic or arachidonic acids) to influence inflammatory processes. Supplementation of the diet of healthy young male subjects with $1.5 \mathrm{~g}$ arachidonic acid per day for 7 weeks resulted in a marked increase in production of $\mathrm{PGE}_{2}$ and $\mathrm{LTB}_{4}$ by endotoxin-stimulated mononuclear cells, although production of TNF- $\alpha$, IL-1 $\beta$ and IL-6 was not significantly altered (12). Thus, increased arachidonic acid intake may result in changes indicative of selectively increased inflammation or inflammatory responses in humans. Supplementation of the diet of healthy elderly subjects with arachidonic acid $(0.7 \mathrm{~g}$ per day in addition to a habitual intake of about $0.15 \mathrm{~g}$ per day) (11) for 12 weeks did not affect endotoxinstimulated production of TNF- $\alpha$, IL- $1 \beta$ or IL- 6 by mononuclear cells and did not alter plasma soluble VCAM-1, ICAM-1 or E-selectin concentrations 
(13). This lack of effect occurred despite incorporation of arachidonic acid into target cells (11). Taken together, these studies suggest that modestly increased intake of arachidonic acid results in incorporation of arachidonic acid into cells involved in inflammatory responses, but that this does not affect production of inflammatory cytokines or shedding of adhesion molecules, although production of inflammatory eicosanoids is increased.

\section{Long-chain n-3 polyunsaturated fatty acids and eicosanoid production}

Increased consumption of the long-chain n-3 PUFAs, EPA and docosahexaenoic acid (DHA), typically found in oily fish and in fish oil liquids and capsules, results in increased proportions of those fatty acids in inflammatory cell-membrane phospholipids, partly at the expense of arachidonic acid (1-7). Thus, there is less substrate available for synthesis of eicosanoids from arachidonic acid. In accordance with this, fish oil supplementation of the human diet has been shown to result in decreased production of $\mathrm{PGE}_{2}(2,5,14), \mathrm{TXB}_{2}$ (5), $\mathrm{LTB}_{4}$ and 5-HETE $(1,3)$ and $\mathrm{LTE}_{4}(15)$ by inflammatory cells. EPA also acts as a substrate for COX and LOX enzymes, so giving rise to a different family of eicosanoids: the 3-series PGs and TXs, and the 5-series LTs and hydroxyeicosapentaenoic acids. Thus, fish oil supplementation of the human diet has been shown to result in increased production of $\mathrm{LTB}_{5}, \mathrm{LTE}_{5}$ and 5-hydroxyeicosapentaenoic acid by inflammatory cells $(1,3,15)$. The functional significance of this is that the mediators formed from EPA are frequently less potent than those formed from arachidonic acid. For example, $\mathrm{LTB}_{5}$ is 10-100-fold less potent as a neutrophil chemotactic agent than $\operatorname{LTB}_{4}(16,17)$, while $\mathrm{PGE}_{3}$ is a less potent inducer than $\mathrm{PGE}_{2}$ of COX-2 gene expression in fibroblasts and of IL-6 production by macrophages (8).

In addition to long-chain n-3 PUFAs modulating the generation of eicosanoids from arachidonic acid and to EPA acting as substrate for the generation of alternative eicosanoids, recent studies have identified a novel group of mediators, termed E-series resolvins, formed from EPA by COX-2 and 5-LOX, that appear to exert anti-inflammatory actions (18). In addition, DHA-derived mediators termed Dseries resolvins, docosatrienes and neuroprotectins, also produced by COX-2 and 5-LOX under some conditions, have been identified and these too appear to be anti-inflammatory and inflammation resolving (18).

\section{Anti-inflammatory effects of $n-3$ polyunsaturated fatty acids other than altered eicosanoid production}

Although their action in antagonizing arachidonic acid metabolism is a key anti-inflammatory effect of n-3 PUFAs, these fatty acids have a number of other anti-inflammatory effects that may occur downstream of altered eicosanoid production or may be independent of this.

Long-chain n-3 PUFAs affect the cell-surface expression of adhesion molecules that are involved in interactions between leukocytes and endothelial cells and that lead to leukocyte infiltration into sites of inflammatory activity. This was first demonstrated by de Caterina et al. (19), who showed that culture of human venous endothelial cells with DHA significantly decreased cytokine-induced surface expression of E-selectin, ICAM-1 and VCAM1 , and impaired the ability of ligand-bearing monocytes to adhere (20). Dietary fish oil decreased expression of ICAM-1 on the surface of murine macrophages (21) and decreased circulating levels of soluble VCAM-1 in elderly subjects (22), although it is not clear whether this represents decreased surface expression of VCAM-1.

Long-chain n-3 PUFAs also affect synthesis of inflammatory cytokines, a process that is regulated in part by $\mathrm{PGE}_{2}$ and 4-series LTs. Cell-culture studies demonstrate that both EPA and DHA can inhibit the production of TNF- $\alpha$ and IL-1 $\beta$ by monocytes, and the production of IL- 6 and IL- 8 by venous endothelial cells $(23,24)$. Fish oil feeding decreased ex vivo production of TNF- $\alpha$, IL- $1 \beta$ and IL- 6 by rodent macrophages $(23,24)$ and decreased circulating concentrations of these cytokines in experimental endotoxaemia in mice (25). Supplementation of the diet of healthy human volunteers with fish oil has been reported to result in decreased ex vivo production of TNF, IL-1 and IL-6 by mononuclear cells $(2,5,26-28)$, although not all studies agree on this point (29).

\section{n-3 Polyunsaturated fatty acids and inflammatory gene expression}

De Caterina et al. (19) demonstrated that the downregulation of VCAM-1 expression on endothelial cells caused by DHA was exerted at the level of 
VCAM-1 gene expression, and that this effect was independent of effects on eicosanoid production and on antioxidant status. Culturing bovine chondrocytes with EPA or DHA markedly decreased cytokine-mediated induction of expression of the COX-2, TNF- $\alpha$ and IL- $1 \alpha$ genes (30). Including EPA or DHA in the culture medium of human osteoarthritic cartilage explants markedly decreased the cytokine-induced up-regulation of expression of the COX-2, IL- $1 \alpha$, IL- $1 \beta$, TNF- $\alpha$, and 5-LOX activating protein and matrix metalloproteinase genes in these cells (31). Inclusion of fish oil in the diet completely abolished mRNA for TNF- $\alpha$, IL- $1 \beta$ and IL-6 in the kidneys of autoimmune diseaseprone mice (32). Feeding mice a fish oil-rich diet significantly decreased the level of IL-1 $\beta$ mRNA in endotoxin- or phorbol ester-stimulated spleen lymphocytes (33); the lower IL-1 $\beta$ mRNA level was due not to accelerated degradation but to impaired synthesis. Fish oil feeding to mice lowered basal and endotoxin-stimulated TNF- $\alpha$ mRNA levels in peritoneal macrophages (34). ICAM-1 mRNA levels were lower in fresh peritoneal macrophages from mice fed fish oil (21).

It is now emerging that n-3 PUFAs may exert their effects on inflammatory gene expression through direct actions on the intracellular signalling pathways that lead to activation of one or more transcription factors such as nuclear factor $-\kappa \mathrm{B}$ $(\mathrm{NF}-\kappa \mathrm{B})$. Studies have shown that n-3 PUFAs can decrease the extent of activation of $N F-\kappa B$ in cultured monocytes, an effect associated with

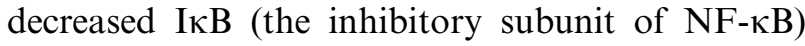
phosphorylation, perhaps due to decreased activation of mitogen-activated protein kinases (35). These observations suggest direct effects of long-

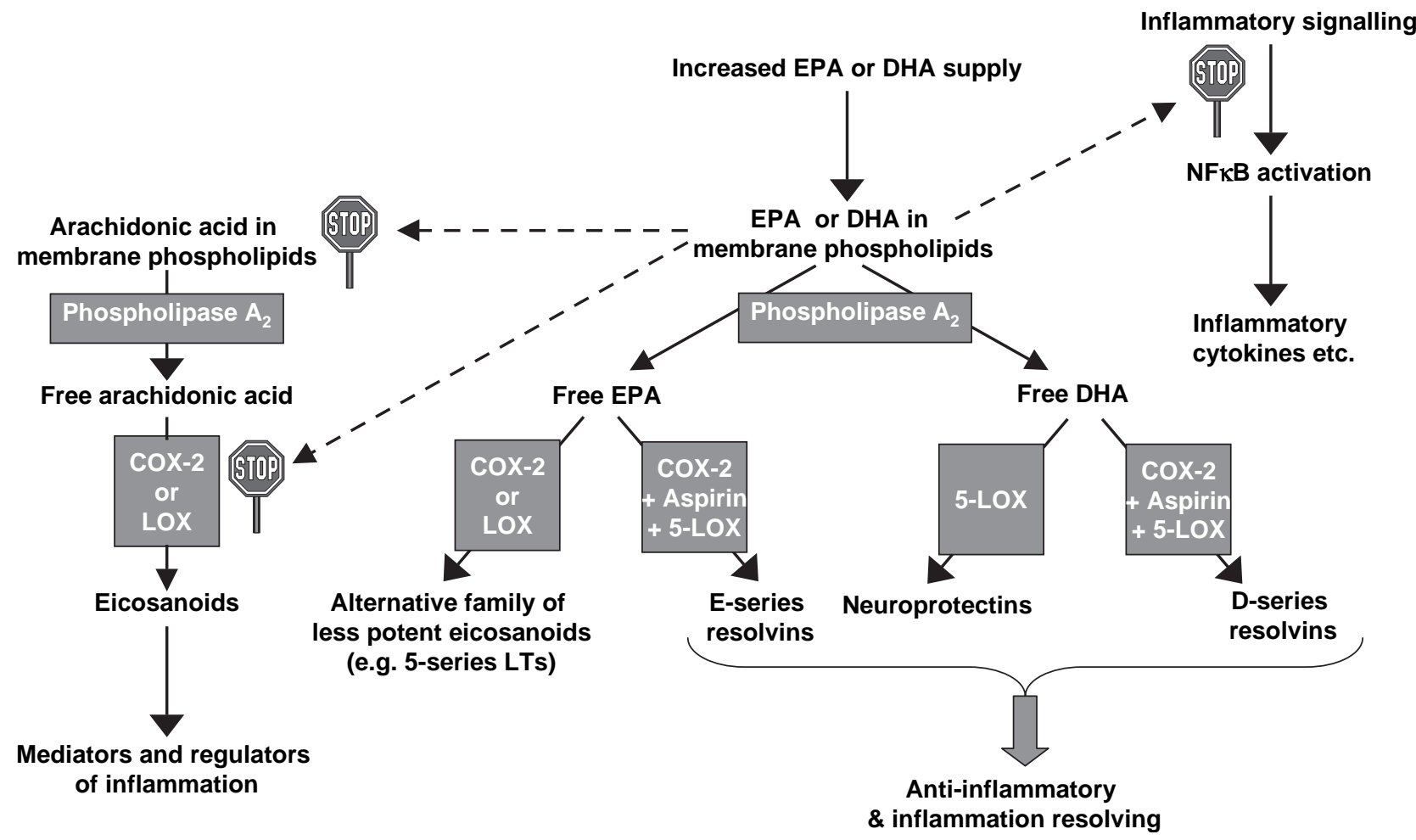

Fig. 2. Mechanisms by which long-chain n-3 polyunsaturated fatty acids exert anti-inflammatory effects. Increased dietary supply of eicosapentaenoic acid (EPA) or docosahexaenoic acid (DHA) results in their incorporation into inflammatory cell membranes partly at the expense of arachidonic acid. EPA and DHA also inhibit arachidonic acid metabolism by cyclooxygenase (COX) and lipoxygenase (LOX) enzymes. Thus, EPA and DHA decrease the production of arachidonic acid-derived eicosanoids. EPA metabolism by COX-2 and 5-LOX enzymes gives rise to eicosanoids that are typically less potent than those formed from arachidonic acid. DHA metabolism by 5-LOX gives rise to anti-inflammatory neuroprotectins. Metabolism of EPA or DHA by COX-2 and 5-LOX in the presence of aspirin gives rise to antiinflammatory resolvins. EPA and DHA also inhibit the signalling pathway, leading to activation of nuclear factor- $\kappa \mathrm{B}(\mathrm{NF}-\kappa \mathrm{B})$, a transcription factor that induces expression of a number of inflammatory genes, including those encoding inflammatory cytokines, adhesion molecules and COX-2. 
chain n-3 fatty acids on inflammatory gene expression via inhibition of activation of the transcription factor $N F-\kappa B$.

\section{Clinical applications of the anti-inflammatory actions of long-chain n-3 polyunsaturated fatty acids}

Inflammation is an overt or a covert component of numerous human diseases and conditions (Table 1). Although the inflammation may afflict different body compartments, one common characteristic of these conditions and diseases is excessive or inappropriate production of inflammatory mediators including eicosanoids and cytokines. The recognition that the long-chain n-3 PUFAs have antiinflammatory actions has led to the ideas that an absolute or relative lack of these fatty acids may contribute causally to inflammatory conditions and that supplementation of the diet of patients with inflammatory diseases may bring about clinical benefit. Supplementation trials with long-chain n-3 PUFAs have been conducted in many of the states listed in Table 1. In many cases these demonstrate anti-inflammatory effects, such as changes in circulating concentrations or ex vivo production of inflammatory mediators. There is evidence of clinical benefit from trials conducted in rheumatoid arthritis, but evidence of clinical efficacy is still lacking in most of the other conditions mentioned (see refs 23, 24, 35 and 36 for further discussion).

\section{$\alpha$-Linolenic acid and inflammation}

Relatively few studies have examined the effect of the precursor n-3 PUFA $\alpha$-linolenic acid on inflammatory outcomes in humans. Caughey et al. (5) reported that $13.7 \mathrm{~g} \alpha$-linolenic acid per day for 4 weeks resulted in a decrease in production of TNF- $\alpha$ and IL-1 $\beta$ by endotoxin-stimulated mononuclear cells by 27 and 30\%, respectively. By comparison, fish oil providing $2.7 \mathrm{~g}$ EPA + DHA per day decreased production of these cytokines by 70 and $78 \%$, respectively (5). Thus, on a grams per day basis long-chain n-3 PUFAs are about nine times more potent than $\alpha$-linolenic acid with respect to this outcome in healthy subjects. Several studies using lower intakes of $\alpha$-linolenic acid than used by Caughey et al. (2-9.5 g per day) did not find antiinflammatory effects (37). Taken together, these data suggest that increasing $\alpha$-linolenic acid intake to $>10 \mathrm{~g}$ per day is required for anti-inflammatory effects to be seen. Even then, the effects will be much more modest than those exerted by longchain n-3 PUFAs (5).

\section{Conclusions}

Long-chain n-3 PUFAs from oily fish and fish oils decrease the production of inflammatory eicosanoids, cytokines and adhesion molecules. They act both directly, by replacing arachidonic acid as an eicosanoid substrate, by inhibiting arachidonic acid metabolism and by giving rise to anti-inflammatory resolvins, and indirectly, by altering the expression of inflammatory genes through effects on transcription factor activation (Fig. 2). Thus, long-chain n-3 PUFAs are potentially useful anti-inflammatory agents and may be of benefit in patients at risk of a variety of acute and chronic inflammatory in settings. The precursor n-3 PUFA, $\alpha$-linolenic acid, is much less potent.

\section{References}

1. Lee TH, Hoover RL, Williams JD, Sperling RI, Ravalese J, Spur BW, et al. Effects of dietary enrichment with eicosapentaenoic acid and docosahexaenoic acid on in vitro neutrophil and monocyte leukotriene generation and neutrophil function. N Engl J Med 1985; 312: 1217-24.

2. Endres S, Ghorbani R, Kelley VE, Georgilis K, Lonnemann G, van der Meer JMW, et al. The effect of dietary supplementation with n-3 polyunsaturated fatty acids on the synthesis of interleukin-1 and tumor necrosis factor by mononuclear cells. N Engl J Med 1989; 320: 265-71.

3. Sperling RI, Benincaso AI, Knoell CT, Larkin JK, Austen KF, Robinson DR. Dietary $\omega-3$ polyunsaturated fatty acids inhibit phosphoinositide formation and chemotaxis in neutrophils. J Clin Invest 1993; 91: 651-60.

4. Gibney MJ, Hunter B. The effects of short- and longterm supplementation with fish oil on the incorporation of n-3 polyunsaturated fatty acids into cells of the immune system in healthy volunteers. Eur J Clin Nutr 1993; 47: 255-9.

5. Caughey GE, Mantzioris E, Gibson RA, Cleland LG, James MG. The effect on human tumor necrosis factor $\alpha$ and interleukin $1 \beta$ production of diets enriched in $n-3$ fatty acids from vegetable oil or fish oil. Am J Clin Nutr 1996; 63: 116-22.

6. Yaqoob P, Pala HS, Cortina-Borja M, Newsholme EA, Calder PC. Encapsulated fish oil enriched in $\alpha$-tocopherol alters plasma phospholipid and mononuclear cell fatty acid compositions but not mononuclear cell functions. Eur J Clin Invest 2000; 30: 260-74.

7. Healy DA, Wallace FA, Miles EA, Calder PC, Newsholme P. The effect of low to moderate amounts of 
dietary fish oil on neutrophil lipid composition and function. Lipids 2000; 35: 763-8.

8. Bagga D, Wang L, Farias-Eisner R, Glaspy JA, Reddy ST. Differential effects of prostaglandin derived from $\omega-6$ and $\omega-3$ polyunsaturated fatty acids on COX-2 expression and IL-6 secretion. Proc Natl Acad Sci USA 2003; 100: 1751-6.

9. Levy BD, Clish CB, Schmidt B, Gronert K, Serhan CN. Lipid mediator class switching during acute inflammation: signals in resolution. Nat Immunol 2001; 2: 612-9.

10. Peterson LD, Jeffery NM, Thies F, Sanderson P, Newsholme EA, Calder PC. Eicosapentaenoic and docosahexaenoic acids alter rat spleen leukocyte fatty acid composition and prostaglandin $\mathrm{E}_{2}$ production but have different effects on lymphocyte functions and cellmediated immunity. Lipids 1998; 33: 171-80.

11. Thies F, Nebe-von-Caron G, Powell JR, Yaqoob P, Newsholme EA, Calder PC. Dietary supplementation with $\gamma$-linolenic acid or fish oil decreases $\mathrm{T}$ lymphocyte proliferation in healthy older humans. J Nutr 2001; 131: 1918-27.

12. Kelley DS, Taylor PC, Nelson GJ, Mackey BE. Arachidonic acid supplementation enhances synthesis of eicosanoids without suppressing immune functions in young healthy men. Lipids 1998; 33: 125-30.

13. Thies F, Miles EA, Nebe-von-Caron G, Powell JR, Hurst TL, Newsholme EA, Calder PC. Influence of dietary supplementation with long chain n-3 or n-6 polyunsaturated fatty acids on blood inflammatory cell populations and functions and on plasma soluble adhesion molecules in healthy adults. Lipids 2001; 36: 1183-93.

14. Trebble TM, Wootton SA, Miles EA, Mullee M, Arden $\mathrm{NK}$, Ballinger $\mathrm{AB}$, et al. Prostaglandin $\mathrm{E}_{2}$ production and T-cell function after fish-oil supplementation: response to antioxidant co-supplementation. Am J Clin Nutr 2003; 78: 376-82.

15. Von Schacky C, Kiefl R, Jendraschak E, Kaminski WE. n-3 Fatty acids and cysteinyl-leukotriene formation in humans in vitro, ex vivo and in vivo. $\mathrm{J}$ Lab Clin Med 1993; 121: 302-9.

16. Goldman DW, Pickett WC, Goetzl EJ. Human neutrophil chemotactic and degranulating activities of leukotriene $\mathrm{B}_{5}\left(\mathrm{LTB}_{5}\right)$ derived from eicosapentaenoic acid. Biochem Biophys Res Commun 1983; 117: 282-8.

17. Lee TH, Mencia-Huerta JM, Shih C, Corey EJ, Lewis RA, Austen KF. Characterization and biologic properties of 5,12-dihydroxy derivatives of eicosapentaenoic acid, including leukotriene- $\mathrm{B}_{5}$ and the double lipoxygenase product. J Biol Chem 1984; 259: 2383-9.

18. Serhan CN, Arita M, Hong S, Gotlinger K. Resolvins, docosatrienes, and neuroprotectins, novel omega-3-derived mediators, and their endogenous aspirin-triggered epimers. Lipids 2004; 39: 1125-32.

19. de Caterina R, Cybulsky MI, Clinton SK, Gimbrone MA, Libby P. The omega-3 fatty acid docosahexaenoate reduces cytokine-induced expression of proatherogenic and proinflammatory proteins in human endothelial cells. Arterioscler Thromb 1994; 14: 1829-36.
20. de Caterina R, Libby P. Control of endothelial leukocyte adhesion molecules by fatty acids. Lipids 1996; 31: S57-63.

21. Miles EA, Wallace FA, Calder PC. Dietary fish oil reduces intercellular adhesion molecule 1 and scavenger receptor expression on murine macrophages. Atherosclerosis 2000; 152: 43-50.

22. Miles EA, Thies F, Wallace FA, Powell JR, Hurst TL, Newsholme EA, Calder PC. Influence of age and dietary fish oil on plasma soluble adhesion molecule concentrations. Clin Sci 2001; 100: 91-100.

23. Calder PC. n-3 Polyunsaturated fatty acids and inflammation: from molecular biology to the clinic. Lipids 2003; 38: 342-52.

24. Calder PC. n-3 Polyunsaturated fatty acids, inflammation, and inflammatory diseases. Am J Clin Nutr 2006; 83: $1505-19$ S.

25. Sadeghi S, Wallace FA, Calder PC. Dietary lipids modify the cytokine response to bacterial lipopolysaccharide in mice. Immunology 1999; 96: 404-10.

26. Meydani SN, Endres S, Woods MM, Goldin BR, Soo C, Morrill-Labrode A, et al. Oral (n-3) fatty acid supplementation suppresses cytokine production and lymphocyte proliferation: comparison between young and older women. J Nutr 1991; 121: 547-55.

27. Abbate R, Gori AM, Martini F, Brunelli T, Filippini M, Francalanci I, et al. n-3 PUFA supplementation, monocyte PCA expression and interleukin-6 production. Prostaglandins Leukot Essent Fatty Acids 1996; 54: 439-44

28. Trebble T, Arden NK, Stroud MA, Wootton SA, Burdge GC, Miles EA, et al. Inhibition of tumour necrosis factor- $\alpha$ and interleukin- 6 production by mononuclear cells following dietary fish-oil supplementation in healthy men and response to antioxidant co-supplementation. Br J Nutr 2003; 90: 405-12.

29. Calder PC. n-3 Polyunsaturated fatty acids, inflammation and immunity: pouring oil on troubled waters or another fishy tale? Nutr Res 2001; 21: 309-41.

30. Curtis CL, Hughes CE, Flannery CR, Little CB, Harwood JL, Caterson B. n-3 Fatty acids specifically modulate catabolic factors involved in articular cartilage degradation. J Biol Chem 2000; 275: 721-4.

31. Curtis CL, Rees SG, Little CB, Flannery CR, Hughes $\mathrm{CE}$, Wilson $\mathrm{C}$, et al. Pathologic indicators of degradation and inflammation in human osteoarthritic cartilage are abrogated by exposure to n-3 fatty acids. Arthritis Rheum 2002; 46: 1544-53.

32. Chandrasekar B, Fernandes G. Decreased pro-inflammatory cytokines and increased antioxidant enzyme gene expression by $\omega-3$ lipids in murine lupus nephritis. Biochem Biophys Res Commun 1994; 200: 893-8.

33. Robinson DR, Urakaze M, Huang R, Taki H, Sugiyama E, Knoell CT, et al. Dietary marine lipids suppress continuous expression of interleukin- $1 \beta$ gene expression. Lipids 1996; 31: S23-31.

34. Renier G, Skamene E, de Sanctis J, Radzioch D. Dietary n-3 polyunsaturated fatty acids prevent the development of atherosclerotic lesions in mice: modulation of 
macrophage secretory activities. Arterioscler Thromb 1993; 13: 1515-24.

35. Calder PC. n-3 Fatty acids, inflammation and immunity - relevance to postsurgical and critically ill patients. Lipids 2004; 39: 1147-61.

36. Kremer JM, ed. Medicinal fatty acids in inflammation. Basel: Birkhauser Verlag; 1998.

37. Burdge GC, Calder PC. Dietary $\alpha$-linolenic acid and health-related outcomes: a metabolic perspective. Nutr Res Rev 2006; 19: 26-52.

\section{Philip C. Calder}

Institute of Human Nutrition School of Medicine University of Southampton Bassett Crescent East

Southampton SO 16 7PX UK

Tel: +442380594223

Fax: +442380594383

E-mail: pcc@soton.ac.uk 